Editoral

\title{
PREVENTION OF CARDIOVASCULAR DISEASE : PLEA FOR A RATIONALE USE OF DIET AND LIPID-LOWERING DRUGS
}

\author{
A.J. Scheen ${ }^{1}$
}

Cardiovascular disease (CVD) is a major public health problem in industrialized countries. Decades of research have established major variable risk factors for the development of CVD, such as smoking, elevated blood pressure, dyslipidaemia, diabetes mellitus, etc. Consideration of all these factors together allows the calculation of individual 10-year absolute cardiovascular risk scores and the identification of those individuals who in priority deserve more rigorous lifestyle and possibly drug interventions (1). Despite several controversies, lowering low-density lipoprotein (LDL) cholesterol is still the emphasis of the prevention of coronary heart disease (CHD) $(1,2)$. To achieve the LDL cholesterol-treatment goals, both diet and drugs, essentially statins, are at the forefront of intervention strategies (3).

The scientific basis for attributing a prominent, but not exclusive, role of dietary cholesterol and saturated fat in the genesis of atherosclerosis and ultimately CHD is buttressed by several lines of evidence that have been gathered over the past 150 years. These are essentially animal experiments, epidemiological correlations, and human nutritional studies. These observations support

${ }^{1}$ Professor of Medicine and Clinical Pharmacology, University of Liège, Division of Diabetes, Nutrition and Metabolic Disorders, Department of Medicine, CHU Sart Tilman, Liège, Belgium (Email : andre.scheen@chu.ulg.ac.be)

Address for correspondence :

A.J. Scheen

Division of Diabetes, Nutrition \& Metabolic Disorders,

Department of Medicine,

CHU Sart Tilman,

B-4000 Liège,

Belgium

Fax : 32-4-3667068

E-mail : Andre.scheen@chu.ulg.ac.be the implementation of nutritional approaches to reduce atherosclerotic CVD. The recent United States National Cholesterol Education Program Adult Treatment panel III (NCEP-ATP III) guidelines (2) translate the most recent scientific data into recommendations for healthcare providers. These include the limitation of fat intake (reduced to $25-35 \%$ of calories), saturated fat (7\% of calories) and dietary cholesterol (less than $200 \mathrm{mg} /$ day), and the achievement and maintenance of ideal body weight. These steps are supported by considerable evidence for efficacy in reducing total and LDL cholesterol levels. However, the reduction that can be achieved in cholesterol by diet therapy seems rather small compared with the marked reduction by lipid-lowering agents. Nevertheless, diet is still important, especially on a population basis, but also in high-risk individuals. Indeed, a lipid-lowering diet can reduce the number of individuals requiring drug therapy and diminish the requested dosage to achieve cholesterol target levels, thus reducing the overall cost of drug therapy. Meanwhile, it may result in an added reduction in cholesterol of $5 \%$ beyond that attained by drug therapy, which could provide at least theoretically an additional $5 \%$ reduction in CHD.

Statins are the most potent cholesterol-lowering drugs : they inhibit hepatic HMG-CoA reductase, reduce liver cholesterol synthesis and overexpress hepatocyte LDL receptors, leading to dose-dependent reductions in total and LDL cholesterol levels up to 50-60\% (as compared to a maximum of 10 to $20 \%$ with diet alone). Statins have proven their efficacy for reducing CVD events in numerous controlled, randomised clinical trials according to Evidence-Based Medicine $(4,5)$. Significant LDL cholesterol reductions of $25 \%$ to $35 \%$ and a decrease in coronary risk of $23 \%$ to $37 \%$ have justified the use of statins for lipid lowering in conjunction with diet therapy in high-risk patients $(4,5)$. Furthermore, the latest research demonstrates that statins 
have additional biological effects (so-called pleiotropic effects) that may partially explain their efficacy in reducing the incidence of CHD beyond what is expected from cholesterol reduction alone (6). Overall, statins have potent LDL cholesterol-lowering effects, as well as favourable effects on multiple nonlipid risk factors for CHD. This constellation of benefits provides a powerful approach for reducing risk of CHD, even in individuals with only modest elevations of total and LDL cholesterol levels, as pointed out by the recent results of the Heart Protection Study (7). Because of their cost effectiveness, high compliance rate, and safety when used appropriately, statins should be considered as the drugs of choice to treat hypercholesterolaemia in highrisk CVD-prone patients (1-5).

The survey of Devroey and Willem published in this issue of the Acta Clinica Belgica (8) aimed at evaluating the diet-induced changes in fasting lipoprotein levels among patients who wait for the reimbursement of a lipid-lowering drug according to the Belgian procedure. Of the patients who finally received lipid-lowering drugs (because total cholesterol level remained higher than 250 $\mathrm{mg} / \mathrm{dl}$ or triglycerides remained higher than $200 \mathrm{mg} / \mathrm{dl}$ after 3 months of diet treatment alone), half were not able to decrease their total cholesterol level with diet before the initiation of the pharmacological treatment. However, no information either on precise diet counselling by the health care provider or on patient's diet compliance was available in this study. Furthermore, it would have been interesting to know the lipid changes in patients (whose proportion is unknown) to whom diet was initially recommended and for whom a lipid-lowering drug was finally not prescribed (presumably because diet was effective enough, at least to decrease the lipid levels below the rather high target levels defined by the Belgian reimbursement system, i.e. total cholesterol < $250 \mathrm{mg} / \mathrm{dl}$ ). Finally, the study concerned a mixed population including subjects in either primary or secondary prevention. One can suspect that the rules of diet and drug prescription may differ between the two subgroups. On the one hand, patients in secondary prevention may have a better response to life-style changes than subjects in primary prevention, because of better compliance. On the other hand, physicians may be more motivated to prescribe statins (rather than fibrates) in the former, very high-risk group because of the well-known demonstration of cardiovascular protection provided by this pharmacological class in the last 10 years, especially in secondary prevention.

The Authors (8) pointed out that cardiovascular risk factors are still not taken into account for the reimbursement of statins in Belgium. Indeed, the only criterion is the maintenance of cholesterol levels above $250 \mathrm{mg} / \mathrm{dl}$ after 3 months of diet therapy, whatever the CVD risk profile. Obviously, Belgian reimbursement criteria are old fashioned and should be adapted urgently for a more rationale use of lipid-lowering drugs in daily practice according to Evidence-Based Medicine (4). Indeed, with the current procedure, numerous patients at very high risk, because of previous cardiovascular events (secondary prevention) or a high-risk cardiovascular profile (primary prevention with an estimated score of cardiovascular events above $20 \%$ in the next 10 years), are not in a position to benefit from statin therapy despite the fact that statins have extensively proven their efficacy and cost-effectiveness in such a population. Interestingly enough, the primary prevention Anglo-Scandinavian Cardiac Outcomes Trial-Lipid Lowering Arm (ASCOTLLA) was performed in individuals who would not have benefit from the reimbursement of lipid-lowering agents according to current Belgian criteria, i.e. hypertensive patients with at least three other cardiovascular risk factors, but with total cholesterol concentrations $250 \mathrm{mg} /$ dl or less (9). Nevertheless, after a median follow-up of 3.3 years, the incidence of non-fatal myocardial infarction and fatal coronary heart disease was reduced by $36 \%$ in patients treated with atorvastatin as compared to the placebo group. Moreover, the rule of imposing a 3 -month diet therapy in patients with recent cardiovascular event may contribute to delay the prescription of an active drug and to increase the risk that such a drug will never be given. Indeed, the EURO-ASPIRE II survey demonstrated that, among individuals from $15 \mathrm{Eu}$ ropean countries, Belgian patients with CHD had the highest cholesterol level and the lowest rate of statin therapy at hospital discharge and 1-2 years later, following a serious CHD event (10). In contrast, with the current Belgian procedure, numerous individuals may receive a lipid-lowering drug because total cholesterol levels remain above $250 \mathrm{mg} / \mathrm{dl}$ although they are not at high risk for cardiovascular disease, especially in the female population. The evidence of any benefit of treating such subjects with lipid-lowering agents is scarce, and such inappropriate use of expensive drugs is waste of money.

Finally, Devroey and Willem made a proposal for new reimbursement criteria in Belgium (8). The main difference as compared to the recommendations of the Second European Joint Task Force (1), endorsed by the Belgian Lipid Club, is the use of the ratio of total cho- 
lesterol to HDL cholesterol instead of total (or LDL) cholesterol. Such change is in agreement with the more recent Joint British recommendations (11), but not with those of the US NCEP-ATP III (2). Even if the role of HDL cholesterol should probably be reconsidered, it seems preferable to wait the new guidelines of the $\mathrm{Eu}-$ ropean Task Force (SCORE) that should be officially presented and published end 2003. The proposal by Devroey and Willem still requests a baseline lipid profile and an initial period of lifestyle changes during three months, even in secondary prevention. This proposal is questionable in view of the recent positive results of the Heart Protection Study (7). This study indeed demonstrated the efficacy of simvastatin in high-risk patients because of previous CVD or diabetes mellitus and microalbuminuria, or hypertension and left ventricular hypertrophy -, independently of the initial total and LDL cholesterol level. Similar results were recently reported in the ASCOT-LLA study (9). Furthermore, evidence from experimental studies and from at least one randomised, placebo-controlled clinical trial (12) supports the use of early and intensive lipid-lowering as part of the initial medical management of patients with acute coronary syndrome. Thus, especially in patients with clinical manifestations of atherosclerosis, current evidence suggests that statins should be prescribed independently of prevailing cholesterol level and of any initial dietary test period. Of course, this does not mean that lifestyle changes are meaningless to reduce CHD and that cholesterol check is needless to verify both efficacy of and compliance to lipid-lowering therapy (13).

In conclusion, a well-balanced optimal use of dietary and pharmacological approaches is needed to reduce CVD in a cost-effective manner. Adequate nutritional advice should be brought to the population at large, while lipid-lowering agents, in addition to diet, should be recommended in an individual approach targeting high-risk patients $(1,2)$. The dynamic duo of diet and lipid-lowering drugs provides the most effective clinical means identified to date for maximally lowering LDL cholesterol levels. However, to impose a period of diet therapy in high-risk patients before reimbursing lipid-lowering agents represents a risk of postponing the most effective treatment and sometimes of never using it !

\section{REFERENCES}

1. Task Force Report. Prevention of coronary heart disease in clinical practice. Recommendations of the Second Joint Task Force of European and other Societies on Coronary Prevention. Eur Heart J 1998; 19: 1434-503.

2. Expert Panel on Detection, Evaluation and Treatment of High Blood Cholesterol in Adults. Executive Summary of the third report of the National Cholesterol Education Program (NCEP) Expert Panel on detection, evaluation, and treatment of high blood cholesterol in adults (Adult Treatment Panel III). JAMA 2001; 285: 2486-97.

3. Clemmer KF, Binkoski AE, Coval SM, Zhao G, Kris-Etherton PM. Diet and drug therapy : a dynamic duo for reducing coronary heart disease risk. Curr Ather Rep 2001; 3: 507-13.

4. Heller FR. Prevention of cardiovascular events by hypolipidemic therapy : evidence-based medicine criteria. Acta Clin Belg 1999 ; 54: 299-301.

5. Kreisberg RA, Oberman A. Lipids and atherosclerosis : lessons learned from randomized controlled trials of lipid lowering and other relevant studies. J Clin Endocrinol Metab 2002; 87: 42337.

6. Bonetti PO, Lerman LO, Napoli C, Lerman A. Statin effects beyond lipid lowering - are they clinically relevant? Eur Heart J 2003; 24: 225-48.

7. Heart Protection Study Collaborative Group. MRC/BHF Heart Protection Study of cholesterol lowering with simvastatin in 20,536 high risk individuals. Lancet 2002; 360: 7-22.

8. Devroey D, Willem B. An analysis of first authorisations for lipid-lowering drugs in Belgium. Acta Clin Belg 2003.

9. Sever PS, Dahlöf B, Poulter NR, et al for the ASCOT investigators. Prevention of coronary and stroke events with atorvastatin in hypertensive patients who have average or lower-than-average cholesterol concentrations, in the Anglo-Scandinavian Cardiac Outcomes Trial- Lipid Lowering Arm (ASCOT-LLA): a multicentre randomised controlled trial. Lancet 2003; 361: 114958.

10. EUROASPIRE II Study Group. Lifestyle and risk factor management and use of drug therapies in coronary patients from 15 countries : principal results from EUROASPIRE II Euro Heart Survey Programme. Eur Heart J 2001; 22: 554-72.

11. British Cardiac Society, British Hyperlipidaemia Association, British Hypertension Society, British Diabetic Association. Joint British recommendations on prevention of coronary heart disease in clinical practice : summary. BMJ 2000; 320: 705-8.

12. Schwartz GG, Olsson AG, Ezekowitz MD et al. Effects of atorvastatin on early recurrent ischemic events in acute coronary syndromes. The MIRACL study : a randomised controlled trial. JAMA 2001; 285: 1711-8.

13. Scheen AJ, Kulbertus H. Prévention cardio-vasculaire par les statines : faut-il encore doser le cholestérol ? Rev Med Liège 2003; 58. 